\title{
3D Imaging of Polymer-based Materials by Laser Scanning Confocal Microscopy
}

\section{Liang}

DuPont Central Research and Development, P. O. Box 80228, Wilmington, DE 198800228

One of the greatest advances in microscopy in the past two decades is the development of laser scanning confocal microscopy (LSCM). [1] The technique built upon many new technologies offers non-destructive three-dimensional imaging capability. In conventional fluorescence microscopy much of the structural detail that could otherwise be resolved is obscured due to the contribution of fluorescence from regions outside of plane of focus. By using a pinhole restricting the collection of light from out-of-focus regions, confocal microscopy provides both significantly higher lateral resolution and possibility to produce 3-D images of the sample without destruction. By scanning in $\mathrm{x}-\mathrm{y}$ plane and moving the microscope stage along the $\mathrm{z}$ direction step by step, a series of optical "sectioning" images can be generated. Towards the end of last century, new multi-photon excitation without pinhole has been added to LSCM to reach deeper penetration, lower UV radiation damage. Therefore, LSCM has been widely used in biological and medical research and development. However, the industrial applications of laser scanning confocal microscopy in material science and technology have been rather limited. [23] Recently, we have used a Zeiss-510 NLO two-photon scanning laser confocal microscope (TPLSCM) to investigate some industrially important polymer-based materials and report here.

Zeiss $510 \mathrm{NLO}$ is composed of Axiovert $100 \mathrm{M}$ inverse microscope, three visible laser lines, and one tunable near-infrared (NIR) Ti:Sapphire laser. The maximum scan resolution is 2048x2048 pixels in digital images. The stage can move a step of as small as $25 \mathrm{~nm}$. Many long-pass or band-pass filters are available for the selection of the interested emission spectrum.

Electron microscopy including transmission and scanning electron microscopy (TEM, SEM), and conventional optical microscopy are very important microstructure characterization techniques to image multi-phase materials, such as $\mathrm{TiO} 2$ particles in a polymer matrix. However, the images obtained directly with these microscopes are all 2-D images since they are taken either from the surface or from two-dimensional projection of the samples. In some special cases, even 3D SEM images of multi-phase materials can be generated after time-consuming sample preparation process, many artifacts may be introduced due to destructive nature ofthese sample preparation techniques. If matrix materials are transparent, LSCM may be a very useful microstructure characterization technique. 3D images of multiphase materials can be obtained without or with little sample preparation in short time. The second phase added in a polymer matrix may be organic (another polymer) or inorganic particles. The size distribution and dispersion of these particles in the polymer are usually important to the physical and chemical properties of the final products. In the past, to study these microstructure parameters, the samples were usually polished and then observed by scanning electron microscopy. However, due to polishing, the remaining parts of particles appear in the images may no longer reflect real size of the particles. Using LSCM, samples can be directly imaged as deep as several hundreds microns from the top surface without sample preparation. Based on digital LSCM 3D images, particle size distribution and dispersion should be adequately obtained. 
This technique can also be used for the 3D topographic reconstruction of surface and the measurements of surface roughness of materials. Although there are other surface measurement techniques, one of important advantages of LSCM technique is its physically nontouching nature, which are important to surface microstructural characterization of many soft materials.

In industry, quantitative measurements are often needed such as size distribution of second phase particles in polymer matrix materials. LSCM images of the samples are all in digital form. Thus, quantitative measurement can be very conveniently conducted based on these $3 \mathrm{D}$ images through image analysis techniques. Further developments of 3D image analysis will over pass many limitations that was imposed by 2D image analysis.

Like many other microstructural characterization techniques, LSCM has its own limitations. First, the resolution is rather still low, just slightly higher than that of optical microscope. Second, LSCM is not suitable for the materials that are non-transparent or contain too many dark pigments. It should be noticed that if loading of the second phase materials are too high, the upper particles would block the light coming from the particles below and they would be invisible in the final 3D images. Thus, it is crucial to use multiple microscopy techniques to solve real industrially important problems.

\section{References:}

[1]. J. Pawley, Editor, The handbook of biological confocal microscopy, IMR Press, Madison, 1989

[2]. Y. Song et al., Macromolecules, 33 (2000) 4478.

[3]. A. E. Ribbe, Trends in Polymer Science, 5 (1997) 333. 